J. Sustain. Wireless Syst.

Vol.01/ No. 02

Pages: 87-102

http://irojournals.com/irosws/

DOI: https://doi.org/10.36548/jsws.2019.2.002

\title{
ENERGY EFFICIENT MULTI-TIER SUSTAINABLE SECURE ROUTING PROTOCOL FOR MOBILE WIRELESS SENSOR NETWORKS
}

\author{
Dr. JENNIFER S RAJ, \\ Department of ECE, Gnanamani College of Technology, \\ Namakal, India \\ Email id: jennifer.raj@gmail.com
}

\author{
Dr. S. Smys, \\ Department of CSE, RVS Technical Campus, \\ Coimbatore, India. \\ Email id: $\underline{\text { smys375@gmail.com }}$
}

\author{
Dr. Abul Bashar, \\ Prince Mohammad Bin Fahd University, \\ ,Kingdom of Saudi Arabia.
}

\begin{abstract}
The advanced progress in wireless communication pays attention on information of more sensitivity to be delivered to its destined end with numerous of restrains, such as reliableness, constancy, response time and safety. The advancements in digital gadgetry, the usage of unwired transceivers and microcontrollers has made viable the integration of sensing and computing device with transceivers and power units to form a single unit called sensor nodes, the enveloping of sensor nodes form wireless sensor nodes. Innumerable existing proposals have aimed at the energy efficient and secure transmission of data but have achieved any one at the cost of the other. Noting down the issues of the existing method this paper proposes an energy efficient multi-tier sustainable secure routing protocol (EEMSR) for mobile wireless sensor nodes which is a cohabit of multi-tier secure routing with an AL (Ant Lion) optimizer (CoMSRALO) along with the prime curve cryptography supported by the routing formed in spherical structure. This better encryption and decryption method takes the edge of power consumption and offers control over the utilization of energy which is difficult to be achieved when dispatching the pack and also avoids the intervention of the encroacher and improves the security and minimizes the deprivation caused due the information lost.
\end{abstract}

Keywords: Wireless sensor network, AL optimizer, multi-secure, spherical structure routing, sustainable, security, power consumption, encryption.

\section{Introduction}

The wireless network is used to provide a communication between two junctions which are not connected through cables. This network enables us to avoid the laying and maintenance cost caused by wired networks and are administered using radio communication. The wireless network which has extended its area from PAN (personal area network) to mobile, in order to have a safer way of communication overcoming the hazards such as interference, absorption, fading, and reflection, moved ahead to an efficient way of transmitting by integrating the sensing and computing units to power supply and transceivers into a single unit called sensor nodes, group of such sensor nodes were known as WSN which is wireless sensor network. The wireless sensor networks are often 
J. Sustain. Wireless Syst.

Vol.01/ No. 02

Pages: $87-102$

http://irojournals.com/irosws/

DOI: https://doi.org/10.36548/jsws.2019.2.002

denoted as spatially distributed entities which are dedicated for keeping track of and memorialize the physical changes happening around in the environment is a most emerging efficient wireless network which consists of base station with numerous mote keeping track of the physical changes such as temperature, sound, pressure and simultaneously pass data through network to location defined. When administered under radio communication this network is usually constructed of any one of the various topologies such as star, tree and mesh, accompanied by its own advantages and disadvantages. This WSNs network types differ based on the environment whether it is above the ground, under the ground, or under the water, based on this the WSN may be terrestrial WSNs, under water WSN, underground WSN, multimedia WSN and mobile WSN. The terrestrial, under water and underground WSN are equipped with limited battery power in sensor nodes, where recharging them seems to be a difficult task and the multimedia WSN possess an high level of energy consumption and in addition the proper and easy delivery of contents requires a high bandwidth, compared to the above three the latter mentioned mobile WSN comes with better and improved coverage with the ability to compute sense, communicate and move on its own to be interacted with physical environment. These WSNs come up with various applications such as area monitoring, military, bodily malfunctioning detection, flack detection, Geological changes etc. Though WSNs covers major areas they also come with certain flaws such as low storage capacity, limited battery power, and heavy utilization of power causing an ineffective data transmission. Though various adaptive grouping techniques, and algorithms enable an efficient power management and communication, the clustering plays a main role in enabling a network to be energy efficient, clustering forms a group by dividing the task into smaller task and map the similar tasks into a group such that tasks in a group are completely similar to its group member compared to the other group. Obviously the groups would contain a group head which would be known as cluster head collects the information from the groups and sends it to the main station. This type of collecting the information's together and transmitting would cause improved energy consumption, the clustering formation can be based on two types one is the grouping member nodes of similar characteristic and grouping the member nodes with different characteristics the former known as homogeneous and latter to be known as heterogeneous. The heterogeneous network mode offers improved reliability, better bandwidth efficiency and increased coverage compared to homogeneous network mode. The clustering formed in different methods though manages to give a better performance and efficient functioning causes a need to monitor with care when used in networks of large scale on the area of energy loss, flaw node detection.

Therefore routing playing an important role in wireless network due to its changing features leads to a need for energy efficient, flaw detecting routing with the capacity to overcome its difficulties.

\subsection{Motivation}

Although many attempts were made to have a better performance, still existed certain draw back which paved the stepping stone for the next evolution. The headway to the have an effective route protocol started with a routing that enabled a multipath. It was built with delay maintenance and reliability constraints but suffered from cost of energy due to its movable and stationary nodes that existed between the origins to base station which were 
J. Sustain. Wireless Syst.

Vol.01/ No. 02

Pages: $87-102$

http://irojournals.com/irosws/

DOI: https://doi.org/10.36548/jsws.2019.2.002

liable to explode. This lead to the next step of usage of a Mac protocol that was incorporated in the system using cross layer scheme offered a better performance at the cost of power consumption the next developed routing which engrafted a linear or a nonlinear programming in it suffered from maintaining the quality of service, to overcome the problem with previous method. Cluster routing which achieved the quality of service with a desired energy consumption was framed, but suffered less safety on data's being transmitted over the network.

The long distance transmission of information suffered many intrusions the methods developed namely multi hop, trust based routing and so on achieved few of the finish lines at the cost of other most of the threats were warm hole, sink hole and HELLO flood which further paved way only to more intrusions, so a better encryption method so as to avoid the above threats were developed based on prime curve cryptography which serves as a guard to the above mentioned menace and even worse threats that turned to be a fear in the long distance transmission.

\subsection{Contribution}

The energy efficient multi-tier sustainable secure routing protocol (EEMSR) for mobile wireless sensor networks proposes a cohabit of multi-tier secure routing with an $\mathrm{AL}$ (Ant Lion) optimizer along with the prime curve cryptography supported by the spherical frame work.

The initial stage starting with the AL optimizer leads to take the edge of power consumption and offers control over the utilization of energy which seems to be difficult when dispatching the pack.

And the later stage uses the prime curve cryptography involving multi-tier supported by the spherical structure to avoid the intervention of the encroacher and enhances the security and minimizes the deprivation caused due the information lost.

\subsection{Organization}

The organization of the remaining paper is as follows 2 shows the related works on different routing methods followed, 3 provides the proposed work, 4 result analysis, based on different parameters such as throughput, energy consumption, network life time, packet delivery rate and delay 5 conclusion.

\section{Related work}

Atallah et.al [1] the paper shows how the drawback of $\mathrm{K}$ relays in transmitting an information and uses a new security co-operative protocol in which source agrees with the destination to share source trusted relay destination 
J. Sustain. Wireless Syst.

Vol.01/ No. 02

Pages: $87-102$

http://irojournals.com/irosws/

DOI: https://doi.org/10.36548/jsws.2019.2.002

link to encode messages so the source uses the source trusted relay destination link to hold the messages in first step and retransmit it in the second step using analog beam forming in order to avoid the silent listeners of transmitted messages, Nair, Suresh P et.al [2] shows how the mobile authentication based the informal random generated number could be made more secure using digital signature and request for GPS co-ordinates. Andrei, Diana, A. X. É. $\mathrm{N}$ et.al [3] the paper shows how radio access controller uses different radio access technology to each radio accessor and checks for the throughput on each radio access controller to make it more energy efficient. Zawodniok, et.al [4] shows how wireless transmission between indefinite number wireless connections is obtained using directed acyclic graph in downstream node and conveyed through the multi-hop, multi-path route to the Internet connected wireless transceiver so as to have an energy efficient transmission in wireless communication Maciej, et.al [5] proposes an energy efficient way of data transmission in mobile communication using multi-hop method between large number of wireless connections to multiple downstream nodes Renold, A et.al[6] the paper proposes a protected data transmission and an equal energy consumption in un wanted wireless sensor network which consists of multiple stationary source nodes and mobile sink in presence of disturbance this is achieved using convex hull algorithm data are transmitted using elliptic curve cryptography and authenticated using ELGamal scheme also ensures to avoid threat by verifying the digital signature and threshold variations using. Alarifi, Abdulaziz et.al[7] the paper shows that the clustering in wireless sensor networks helps in achieving the quality of network by controlling energy consumption and improved data gathering accuracy. The clustering method involves a certain new adaptive technique so as to achieve the desired performance Hayajneh, Thaier et al [8] the paper ensures a fog assisted wireless body network helps in improved and efficient monitoring remote patients and also enables in achieving reduced delay and protection from harmful intrusions by channel assignment, gateway association and a new delay and energy aware routing network. Roy et.al[9] the paper shows how the energy consumption in wireless body area networks are reduced using two hop cost based energy efficient routing protocol Jahir et al [10] shows how mobile ad-hoc network achieves a proper network with improved delay and reduced power consumption until proper communication is established in disaster area network Qi, Xiaohui et.al [11] paper shows how reliable results of general communication can be achieved using a Rayleigh fading and evaluates the reliability security and secrecy energy efficiency performance in communication networks Selvi, M et.al [12] the paper uses an new trust based routing algorithm so as to provide a reliable military communication and checks for the dangerous intrusions using trust score evolution. Mirjalili, Seyedali et.al [13] proposes the Antlion optimizer which is inspired by the antlion hunting nature, this process enables the proposed to have a best cluster head to be selected from clusters. Jolly, et.al [14] uses a cryptography management protocol to provide a secured transmission using similar key generated for encryption and decryption purpose. Yick, et.al [15] it gives a survey on different types of sensors and their performance measures under various aspects. Latré, Benoît, et.al [16] gives an overview of existing network architure and also the different wireless body network sensor nodes available and their involvement in patient monitoring, their performance are measure under different levels. Abbasi et.al [17] compares the clustering methodology base on convergence rate, cluster stableness, clustering overlap, awareness on location and support for 
J. Sustain. Wireless Syst.

Vol.01/ No. 02

Pages: $87-102$

http://irojournals.com/irosws/

DOI: https://doi.org/10.36548/jsws.2019.2.002

node mobility and gives insight on their, objectives, features and complexity etc. Chen et.al [18] gives a arrangement with ball grid array forming an separation between signal objectives and packaging substrate

\section{Proposed work}

The global nature of worldwide system of networks makes it more difficult to rationalize the managing of the system and operation. So this would be made possible with the nature of organization that is hierarchical which allows the group of systems connected with one another at each level organized into multiple levels. The Multi-tier architecture which is an approach of hierarchical architecture segregates the application into different tier that is into layered physical units and forms a pliable and utile application, mostly probably used multi-tier is three tier architecture which has its top most to be user interacted layer and middle one to a logical processor and the least one to be a data storage and access layer that can be updated or superseded. Thus enabling an optimized resource use, maximized throughput and minimized response by offering a better routing allowing a more secure transmission.

The proposed paper energy efficient multi-tier sustainable secure routing protocol for mobile wireless sensor networks propounds an effective approach cohabit of multi-tier secure routing with an AL(Ant Lion) optimizer (CoMSR-ALO) which starts with the selection of nodes and formation of clusters based on repetitive refinement method. Making repetitive computations the members to be added into the clusters are allocated to the nearest vertex and vertex are recomputed until the proper grouping is formed the cluster head selection is done using the AL optimizer and the data transmission is enabled using a multi-tier secure routing protocol overcoming the hazards of the previous methods discussed above, the FIG 1 show the flow chart of the proposed 
J. Sustain. Wireless Syst.

Vol.01/ No. 02

Pages: $87-102$

http://irojournals.com/irosws/

DOI: https://doi.org/10.36548/jsws.2019.2.002

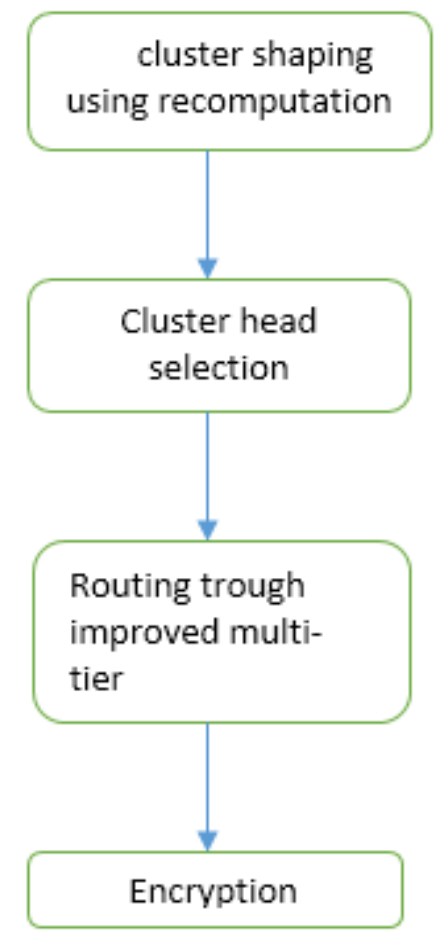

Figure 1 flow chart of the proposed

\subsection{Cluster Shaping on the grounds of AL Optimization}

\section{(a) Cluster Shaping}

Cluster formation enables an easy mode of transmission than that compared to a transmission done on a single standalone device. This cluster shaping as told above is formed using a repetitive refinement method. This does the grouping of data points by engaging them to the nearest vertex on repetitive computations. Vertex decision is done using the chebyshev distance assessment method and the distance between the mote are calculated and used in cluster shaping this is done using the following equation (1)

$$
\operatorname{ND}(x, y)=\operatorname{Maxi}(|x i-y i|)
$$

Where ND stands for the distance between nodes calculated based on chebyshev assessment and this equals to the limits of

$$
\lim _{k=\infty}\left(\sum_{i=1}^{n}|x i-y i|^{\wedge} k\right)^{\wedge} 1 / k
$$


J. Sustain. Wireless Syst.

Vol.01/ No. 02

Pages: $87-102$

http://irojournals.com/irosws/

DOI: https://doi.org/10.36548/jsws.2019.2.002

Under this metric, a sphere of radius $r$, which is the set of points with Chebyshev distance $r$ from a center point, is a square and its sides have the length $2 \mathrm{r}$ and are parallel to the coordinate axe. A consistent node is obtained by calculating the root mean square deviation given by equation (3)

$$
\mathrm{R}=1 / \mathrm{N}\left[\text { sum of square }-\left(\frac{\text { sum }^{2}}{\mathrm{~N}}\right)\right]
$$

Where $\mathrm{N}$ stands for the total number of nodes and the sum of square represents the square of sum of all the nodes gives the root mean square deviation $(\mathrm{R})$. Requirement for the consistent node requires the calculation of also mean given by equation (4)

$$
\bar{X}=1 / \mathrm{n} \quad \sum_{\mathrm{i}=1}^{\mathrm{n}} \mathrm{xi}
$$

Finally the uniformity of the nodes factor is determined using equation (5)

$$
\mathrm{NC}=(\mathrm{C}-\overline{\mathrm{X}}) / \mathrm{R}
$$

Where NC stands for the consistency amount of nodes and c symbolizes the value of the device.

The preliminary vertexes are assessed using the above equations and the cluster is shaped using the following equation (6)

$$
\mathrm{D}_{\mathrm{ij}}^{\mathrm{csd}}=\sqrt[\lambda]{\sum_{\mathrm{k}=1}^{\mathrm{r}}\left|\mathrm{C}_{\mathrm{ij}}-\mathrm{C}_{\mathrm{ij}}\right| \lambda}
$$

So the cluster is obtained using the vertex assessment and the distance calculation method.

The next stride leads us into the election of the cluster head on the grounds of AL optimization

\section{(b) Cluster Head Selection}

As the name suggests the ALO lampoons the insightful characteristics of antlion uses to catch its prey and the based on the survival of the fittest concept the best one is selected the same is now involved here to bring out the best as cluster head.

The optimization initially starts with the forging of the motility of the ants (nodes) by following

$$
M(t)=\{0, \operatorname{Cs}(2 m(t 1)-1), \operatorname{Cs}(2 m(t 2)), \ldots \ldots \ldots \ldots . . . C s(2 m(t n)-1)\}
$$

Where Cs represents the Cumulative summation, and $\mathrm{n}$ represents the maximal number of positions and $\mathrm{m}(\mathrm{t})$ shows the absolute value which is 0 or 1 based on the movement of ants (data points).

The 
J. Sustain. Wireless Syst.

Vol.01/ No. 02

Pages: $87-102$

http://irojournals.com/irosws/

DOI: https://doi.org/10.36548/jsws.2019.2.002

The optimized position saved during the randomized walk of the ants are given as in (8)

$$
\operatorname{Snp}=\left[\begin{array}{ccc}
S_{1,1} & S_{1,2} \ldots & S_{1, p} \\
\vdots & \vdots & \vdots \\
S_{n, 1} & S_{n, 2} & S_{n, p}
\end{array}\right]
$$

Where Snp gives the locale of the ant (node points) and p represents the number of variables and the locale of the each ant (data point) is determined repetitively and their fitness value is saved, however the updated positioning of the ants would be determined by fixing a minimum to maximum range for the ant to move and it is regulated by (9)

$$
\text { Ait }=\frac{\left(A_{i}^{t}-u_{i}\right) *\left(v_{i}-C_{i}^{t}\right)}{\left(v_{i}^{t}-u_{i}\right)}+C i
$$

Where ui gives the minimum movement and vi gives the maximum movement Vit maximum value obtained in tth number of iteration and cit represents the minimum value obtained in th repetition this assures the randomized movement from a minimum to maximum range

To proceed with, the position and fitness of the antlions are also calculated using the below given equation

$$
\mathrm{Sal}=\left[\begin{array}{ccc}
\mathrm{Sl}_{1,1} & \cdots & \mathrm{Sl}_{1, \mathrm{p}} \\
\vdots & \vdots & \vdots \\
\mathrm{Sl}_{\mathrm{n}, 1} & \cdots & \mathrm{Sl}_{\mathrm{n}, \mathrm{p}}
\end{array}\right]
$$

The fitness measurement of the antlions are obtained and recorded in (10)

$$
\mathrm{Sfal}=\left[\begin{array}{ccc}
\mathrm{f}\left[\left(\mathrm{Sl}_{1,1}\right.\right. & \cdots & \left.\left.\mathrm{Sl}_{1, \mathrm{p}}\right)\right] \\
\vdots & \vdots & \vdots \\
\mathrm{f}\left[\left(\mathrm{S}_{\mathrm{n}, 1}\right.\right. & \cdots & \left.\mathrm{S}_{\mathrm{n}, \mathrm{p}}\right)
\end{array}\right]
$$

The fitness values are repeatedly calculated and the fittest antlion that affects the movement of maximum number of the prey is found and movement of the ant around the fittest is given by

$$
\text { Sit }=(\text { Rat }+ \text { Ret }) / 2
$$

The Rat the random movement of the ants in th repetition and the random movement around the fittest is represented by Ret .

And the fittest is found using

$$
\text { Slit }=\text { Sit }, \text { If } \mathrm{f}(\text { Sit })>f(\text { Slit })
$$

The selection of the clusters lead to the next object of security which is determined using an enhanced multi-tier routing protocol. 
J. Sustain. Wireless Syst.

Vol.01/ No. 02

Pages: $87-102$

http://irojournals.com/irosws/

DOI: https://doi.org/10.36548/jsws.2019.2.002

\subsection{Energy Efficient Multi-tier Sustainable secure routing protocol}

Taking into consideration the indemnity of information on network, the transmission starts with converting the plain text in to a different form by the process called encryption and being converted into its original form by the process of decrypting using an asymmetric cryptography technique which is based on public key encryption proves to be more secure than the prevailing symmetric cryptography methods. Here an energy efficient multi-tier sustainable secure routing protocol is used to safeguard the information using the PCC prime curve cryptography which generates a public key and private key that are dissimilar for the encryption and decryption purpose respectively. The network structure in Fig 2 gives the picture of proposed. This is done by set of values agreeing with two in degree of two in one of the variables and three in other and found by satisfying the equation

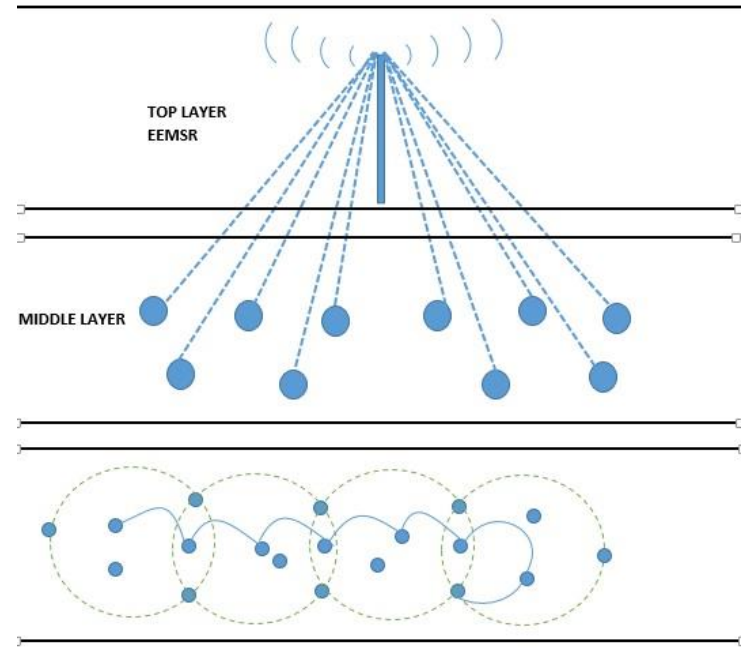

LOWER LAYER (COMSR-ALO)

Fig 2

$$
\mathrm{X} 2=\mathrm{A} 3+\mathrm{CA}+\mathrm{B}
$$

The different values of A, B, C results in different prime curve, commonly used over finite fields over the fields F, it is designated as

$$
\mathrm{X} 2|\mathrm{P}|=(\mathrm{A} 3+\mathrm{CA}+\mathrm{B})|\mathrm{P}|, \mathrm{P} \text { is prime }
$$

The public key is obtained using a random selection of prime number and continuously multiplying it with itself a concealed number of times

$$
\mathrm{PK}=(\mathrm{r} * \mathrm{r}) \mathrm{m}
$$


J. Sustain. Wireless Syst.

Vol.01/ No. 02

Pages: $87-102$

http://irojournals.com/irosws/

DOI: https://doi.org/10.36548/jsws.2019.2.002

PK denotes the public key and $r$ denotes random generated number and $m$ denotes concealed number of times. The determination of the private key is done by (17)

$$
\mathrm{PR}=\sum_{\mathrm{i}=0}^{\mathrm{n}} \mathrm{y}_{\mathrm{i}} \mathrm{j}_{\mathrm{i}}
$$

The information is converted into a machine usable form and reconverted in a human understandable form without any loss by generation of small keys which consume low power

For the encrypted data's are transmitted effectively using a spherical reticulum path to achieve a better conduct, better quality of service and increased life span.

The source to base station transmission is done in a spherical structure. By initially computing the geographical location and extending communication to neighboring node that satisfies the condition $\operatorname{Tr}>2 \mathrm{l} \sqrt{2}$. This route is further extended using the conversion of Cartesian co-ordinates (x, y, and $\mathrm{z}$ ) into spherical co-ordinates $(\mathrm{Q}, \propto, \mathrm{Rd}$ where Rd representing the distance from the center of the circumference is given by

$$
\operatorname{Rd}=\sqrt{\mathrm{x}^{2}+\mathrm{y}^{2}+\mathrm{z}^{2}} \quad \text { where } \operatorname{Rd} \epsilon(0, \infty)
$$

Where $Q$ representing the highest point is given by

$$
Q=\arccos \frac{\mathrm{z}}{\sqrt{\mathrm{x}^{2}+\mathrm{y}^{2}+\mathrm{z}^{2}}}=\arccos \frac{\mathrm{z}}{\mathrm{R}_{\mathrm{d}}}, \boldsymbol{Q} \in(0, \Pi)
$$

Where $\propto$ representing the inverse tangent is given by

$$
\propto=\arctan \frac{\mathrm{y}}{\mathrm{x}}, \propto \in(0,2 \Pi)
$$

And the Cartesian co-ordinates (x, y, and $\mathrm{z}$ ) are determined using

$$
\begin{gathered}
x=R d \sin \varphi \cos \propto \\
y=R d \sin \varphi \sin \propto \\
z=R d \cos Q
\end{gathered}
$$

The $\propto$ is represented by xy plane and the $Q$ represented by yz plane. The position of a point in the spherical coordinate is given by

$$
\mathrm{R}=\mathrm{Rd} \widehat{\mathrm{R}} \mathrm{d}
$$

The information in encrypted form is to be transmitted in a spherical structure and for this purpose the sensor nodes are dispersed in spherical manner and the determined range enables the routing up to the end point between the nodes that poses utmost energy. The spherical routing is formed by the angular position verification of a node made by the preceding and the proceeding nodes and the connection for the data transmission is established allowing a 
J. Sustain. Wireless Syst.

Vol.01/ No. 02

Pages: 87-102

http://irojournals.com/irosws/

DOI: https://doi.org/10.36548/jsws.2019.2.002

fast, flexible, low cost, low power consuming transmission with an increased life span, with a savings of memory and bandwidth thus causing an energy efficient transmission

\section{Result Analysis}

The result evaluation is done based on the requirements such as packet delivery ratio, throughput, energy consumption, network life span and delay. The proposed work is executed using a network simulator with its repetitions countable to 200. The parameters set for simulation are explained in table 1.

\section{Parameter and value table 1}

\begin{tabular}{|c|c|}
\hline parameter & Value \\
\hline Simulation time & 100 seconds \\
\hline Allotted area & $40 \mathrm{~m} * 2500 \mathrm{~m}$ \\
\hline Number of nodes & 500 \\
\hline Packet size & 1024 \\
\hline Packet data rate & 1 packet /second \\
\hline Initial Energy & 100 joules \\
\end{tabular}

The performance metrics are compared with existing low energy adaptive clustering hierarchy and decentralized energy efficient cluster propagation protocol. The number of nodes used measure in the range from 100 to 500 forming maximum number of clusters and then proceeded with the cluster head selection and routing is performed in a spherical manner

\section{(a) Throughput $\left(\mathbf{T}_{P}\right)$ calculation}

Throughput is the maximum rate at which something can be processed in terms of communication networks throughput is defined as the rate of successful transfer of messages over channel.it is usually measured in bits per second. The throughput is usually used to determine the performance measure.

$\mathrm{TP}=$ amount of data transferred /time given 
J. Sustain. Wireless Syst.

Vol.01/ No. 02

Pages: $87-102$

http://irojournals.com/irosws/

DOI: https://doi.org/10.36548/jsws.2019.2.002

The throughput analysis shown in the graph in Fig 3 gives the clear picture of how the proposed method becomes more efficient than the existing low energy adaptive clustering hierarchy and decentralized energy efficient cluster propagation protocol the proposed is executed under varying set of energy levels in order to prove its efficiency. The graph for the throughput measurement for the number of nodes proves to have an improved throughput.

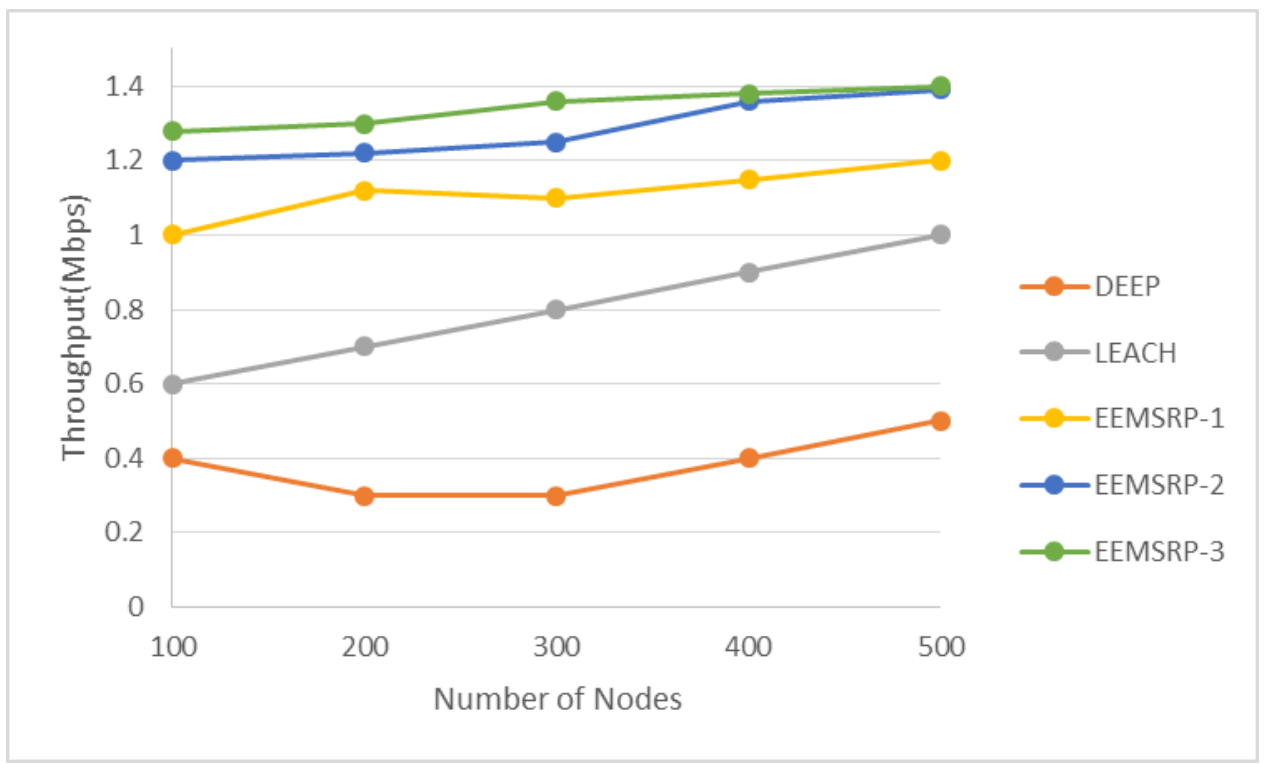

Fig 3

\section{(b) Packet Delivery Rate( $\left.\mathbf{P}_{\mathrm{DR}}\right)$}

Packet delivery rate is defined as the number of successful reception of packets to total number of packets send from the source node

$\mathrm{PDR}=$ number of succesful packets delivered /number of packets sent

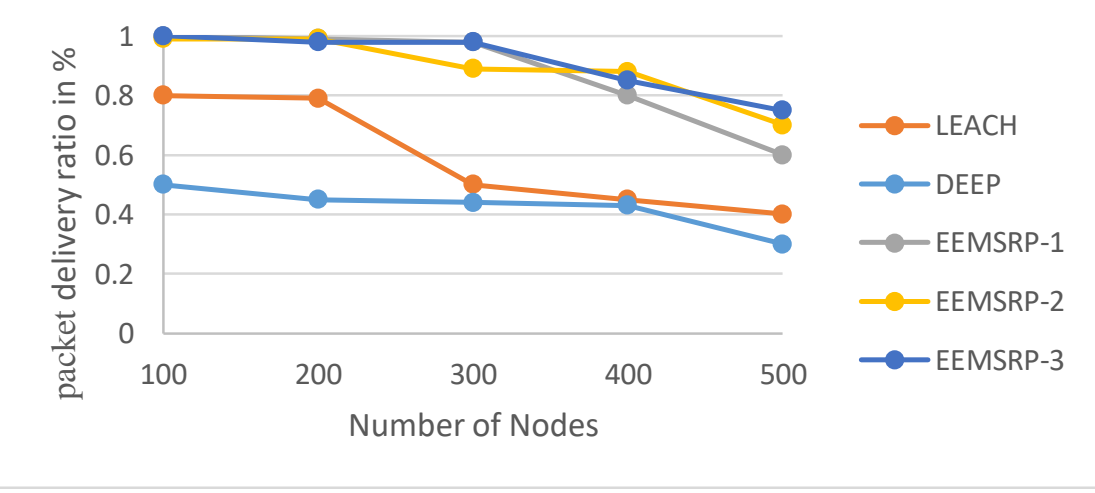


J. Sustain. Wireless Syst.

Vol.01/ No. 02

Pages: $87-102$

http://irojournals.com/irosws/

DOI: https://doi.org/10.36548/jsws.2019.2.002

\section{Fig 4}

Here above result in graph in Fig 4 shows the repeated computations with different set of values for the proposed method has a higher packet delivery ratio when compared to the other two methods thus achieving the maximum delivery rate. Compared to the previously existing method.

\section{(c). Energy Consumption}

The energy consumption being the many criteria to be concentrated on wireless sensor network. The proposed energy efficient multi-tier secure routing protocol used proves an energy efficient transmission compared to the low energy adopted clustering hierarchy and decentralized energy efficient cluster protocol, under different conditions and different computational values.

The energy consumption is calculated using the total amount of transmitted energy multiplied by the number of nodes and added to the receiving energy

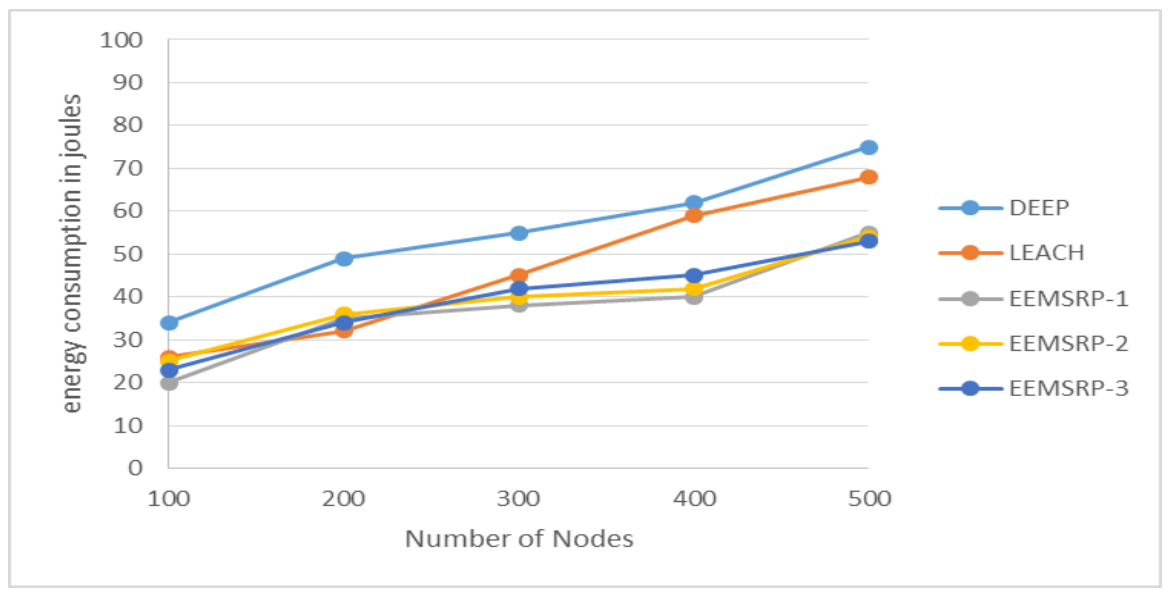

Fig 5

Is given by

Energy consumption $=($ transmitted energy $*$ number of nodes $)+$ received energy

Initially though the energy consumption seems to lower for the previous methods in the later values it takes drastic increase which makes it less energy efficient compared to the proposed is shown in graph in Fig 5.

\section{(d) Network life time}


J. Sustain. Wireless Syst.

Vol.01/ No. 02

Pages: $87-102$

http://irojournals.com/irosws/

DOI: https://doi.org/10.36548/jsws.2019.2.002

The life time of the network is decided by number of alive nodes over time. The maximum number of alive nodes proves that the network has an increased life span.

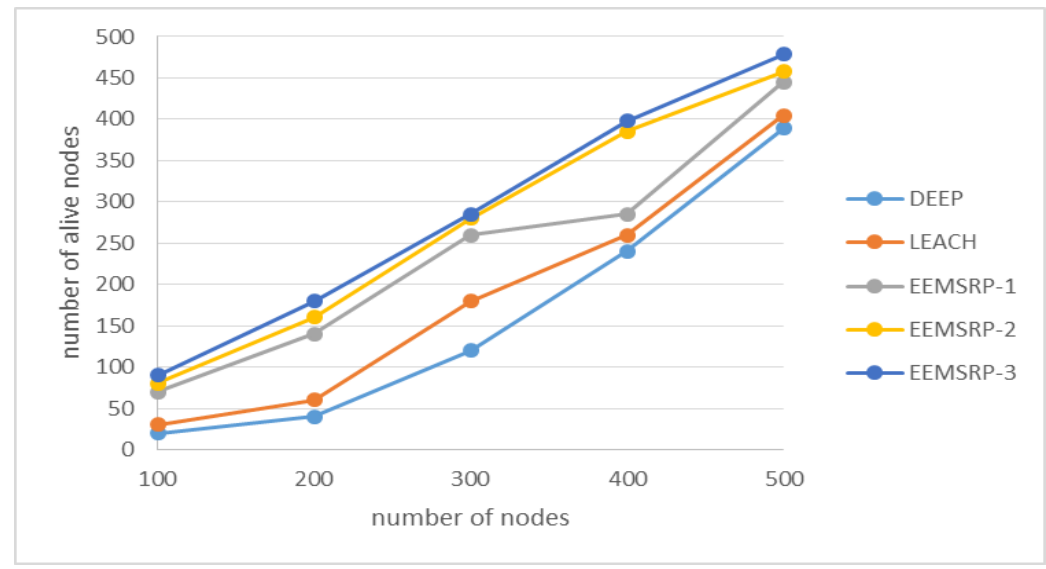

Fig 6

The number of data packets dropped in proposed method is low compared to the other two methods in the result analysis shown in Fig 6 thus proving that the life time of the energy efficient multi-tier secure routing protocol is longer compared to the other.

\section{(e) Delay Calculation}

The delay measures are calculated using the maximum number of packets delivered in given time.

Delay in transmission $=$ maximum number of packets delivered $/$ time 
J. Sustain. Wireless Syst.

Vol.01/ No. 02

Pages: $87-102$

http://irojournals.com/irosws/

DOI: https://doi.org/10.36548/jsws.2019.2.002

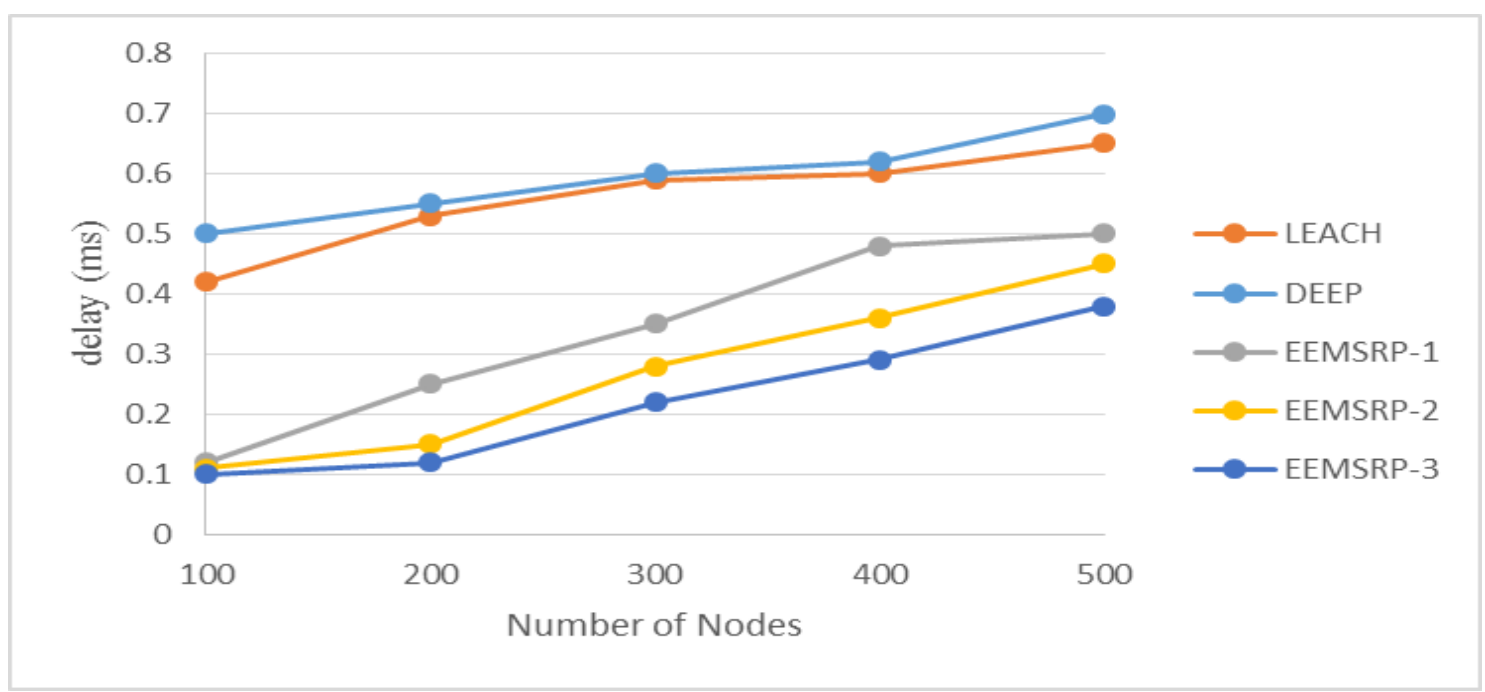

Fig 7

The graph above in Fig 7 shows the maximum number of transmission taken place in given time for the proposed and compared with the existing method the existing proves to have higher delay which might result in lesser number of data transmission in given time.

\section{Conclusion}

The objective of the paper is to achieve a highly secured fast and flexible transmission at low cost and low power consumption. The cohabit of energy efficient multi-tier sustainable secure routing protocol with AL optimizer allows to have highly fittest cluster selected as cluster head and ensures a invulnerable transmission by means of prime curve cryptography on hierarchical order thus allowing an improved energy consumption and secured transmission. The performance measure of the energy efficient multi-tier secure routing protocol on the bases of throughput, delay, energy consumption, network life time and packet data rate are calculated under different levels and repeated computations and compared with the previous existing method and thus proved to be efficient and secured.

\section{References}

[1] Atallah, Michael, and Georges Kaddoum. "Design and Performance Analysis of Secure Multicasting Cooperative Protocol for Wireless Sensor Network Applications." arXiv preprint arXiv:1902.07345 (2019).

[2] Nair, Suresh P., and Semyon Mizikovsky. "Verification of cell authenticity in a wireless network using a system query." U.S. Patent Application 10/200,861, filed February 5, 2019.

[3] Andrei, Diana, A. X. É. N. Rasmus, Elisabeth Hansson, and Stefan Johansson. "System and method for wireless 
J. Sustain. Wireless Syst.

Vol.01/ No. 02

Pages: 87-102

http://irojournals.com/irosws/

DOI: https://doi.org/10.36548/jsws.2019.2.002

network access control." U.S. Patent Application 10/194,376, filed January 29, 2019.

[4] Zawodniok, Maciej, Ivan G. Guardiola, David Bateman, Aaron Phillips, Arul Mathi Maran, and Nathan Daniel Price. "Multi-path wireless mesh networks." U.S. Patent Application 10/182,385, filed January 15, 2019.

[5] Zawodniok, Maciej, Ivan G. Guardiola, David Bateman, Aaron Phillips, Arul Mathi Maran, and Nathan Daniel Price. "Multi-path wireless mesh networks." U.S. Patent Application 14/299,723, filed January 15, 2019.

[6] Renold, A. Pravin, and A. Balaji Ganesh. "Energy efficient secure data collection with path-constrained mobile sink in duty-cycled unattended wireless sensor network." Pervasive and Mobile Computing (2019).

[7] Alarifi, Abdulaziz, and Amr Tolba. "Optimizing the network energy of cloud assisted internet of things by using the adaptive neural learning approach in wireless sensor networks." Computers in Industry 106 (2019): 133-141.

[8] Hayajneh, Thaier, Kristen Griggs, Muhammad Imran, and Bassam J. Mohd. "Secure and efficient data delivery for fogassisted wireless body area networks." Peer-to-Peer Networking and Applications (2019): 1-19.

[9] Roy, Moumita, Chandreyee Chowdhury, and Nauman Aslam. "Designing an energy efficient WBAN routing protocol." In 2017 9th International Conference on Communication Systems and Networks (COMSNETS), pp. 298-305. IEEE, 2017.

[10] Jahir, Yasmin, Mohammed Atiquzzaman, Hazem Refai, Anirudh Paranjothi, and Peter G. LoPresti. "Routing protocols and architecture for Disaster Area Network: A survey." Ad Hoc Networks 82 (2019): 1-14.

[11] Qi, Xiaohui, Bin Li, Zheng Chu, Kaizhi Huang, Hongbin Chen, and Zesong Fei. "Secrecy energy efficiency performance in communication networks with mobile sinks." Physical Communication 32 (2019): 41-49.

[12] Selvi, M., K. Thangaramya, Sannasi Ganapathy, K. Kulothungan, H. Khannah Nehemiah, and A. Kannan. "An Energy Aware Trust Based Secure Routing Algorithm for Effective Communication in Wireless Sensor Networks." Wireless Personal Communications (2019): 1-16.

[13] Mirjalili, Seyedali. "The ant lion optimizer." Advances in Engineering Software 83 (2015): 80-98.

[14] Jolly, Gaurav, Pallavi Kokate, and Mohamed Younis. "A low-energy key management protocol for wireless sensor networks." In null, p. 335. IEEE, 2003.

[15] Yick, Jennifer, Biswanath Mukherjee, and Dipak Ghosal. "Wireless sensor network survey." Computer networks 52, no. 12 (2008): 2292-2330.

[16] Latré, Benoît, Bart Braem, Ingrid Moerman, Chris Blondia, and Piet Demeester. "A survey on wireless body area networks." Wireless Networks 17, no. 1 (2011): 1-18.

[17] Abbasi, Ameer Ahmed, and Mohamed Younis. "A survey on clustering algorithms for wireless sensor networks." Computer communications 30, no. 14-15 (2007): 2826-2841.

[18] Chen, Howard E., David Viveiros, Russ Alan REISNER, and Robert Francis Darveaux. "Signal isolation for module with ball grid array." U.S. Patent Application 15/883,294, filed February 7, 2019. 